\title{
A Decision-making method for the Application of Pavement Repair Materials Based on Stackelberg Model
}

\author{
Gongliang Zhang', Donghang Yu' ${ }^{1}$, Ronghui Zhang ${ }^{2 *}$ \\ ${ }^{1}$ School of Management, Zhongkai University of Agriculture and Engineering, Guangdong, China \\ ${ }^{2}$ School of Construction, Guangdong Polytechnic College, Zhaoqing, Guangdong, China \\ *Corresponding Author.
}

\begin{abstract}
Highway repair cost mainly depends on the toll income, which in turn is associated with the traffic volume. Scientifically making decision for the application of repair materials is within the reasonable planning of managers regarding road maintenance scale and funds. For exploring effective strategy specific to the highway maintenance plan, the paper focuses on integrating the utility factor of traveler into the highway repair decision. Relevant problems are introduced, together with the establishment of the Stackelberg model. Considering the sensitivity analysis, the heuristic algorithm is employed specific to the traffic volume N. The paper solves the decision-making model with regard to the two expressways' maintenance plan, as well as derives corresponding strategies. At last, the paper concludes, based on analyses, the general process regarding the maintenance decisions of multiple highways with the traffic volume $N$, assisting highway operators in making effective decisions.
\end{abstract}

Keywords: Highway pavement, Application of repair Materials, Repair decision, Game theory, Sensitivity analysis

\section{Introduction}

The development of maintenance plans plays a pivotal role in the daily work of the maintenance department, which is closely related to the economic benefits of expressway operators. The highway maintenance plan includes road maintenance fee income and expenditure plan, project plan, etc. The specific work of the project maintenance plan is the road large and medium repair plan, road minor repair and maintenance plan, etc. ${ }^{[1]}$. In toll roads, the total annual income is based on the traffic volume. The larger the traffic volume, the greater the return. Although the traffic volume causes a certain damage to the road surface, which increases the cost of maintenance, the total annual income increases and the maintenance funds with protection, the roads can be arranged in accordance with the requirements of the regulations. With less traffic and less toll income, it is not enough to maintain the normal maintenance of the road, but the road is seriously damaged and the number of users on the road is less ${ }^{[2]}$. Therefore, on the basis of stable maintenance of traffic flow, reasonable arrangement of large and medium-sized repair plans is a measure to improve the economic efficiency of enterprises, and also a problem that decision makers need to consider.

The maintenance plan decision of the custody department and the path choice of the traveler form a game relationship. For business operators with multiple roads in an area, the distribution of traffic on different lines will affect the total revenue and maintenance costs of the management ${ }^{[2]}$. However, whether the maintenance work is in place and whether the road surface is in good condition directly affects the traveler's driving effectiveness and cost, as well as the choice of roads ${ }^{[3]}$. Therefore, in this game problem, the game subject is the management department and the traveler. The management department can maximize the payment (the difference between income and cost) by adjusting the large and medium-sized maintenance plan (size, time, location). The traveler can also choose the travel route according to the road conditions to obtain the maximum payment (driving utility and the difference in cost).

The decision of highway maintenance plan is to use the maintenance management funds reasonably, and invest the limited maintenance funds into the most needed maintenance road sections to maximize the maintenance benefits, so as to ensure the lowest life cycle cost of the road and ensure the most comprehensive network service level. Excellent purpose ${ }^{[4]}$. With the development of the expressway network, highway operating companies usually

ISSN: 0010-8189

www.converter-magazine.info 
manage the maintenance of multiple expressways at the same time. Under the premise of limited maintenance funds, how to rationally allocate maintenance funds to improve the economic efficiency of enterprises is a problem that decision makers need to consider. However, there is a lack of existing research to explore the issue of maintenance plans for the simultaneous management of multiple highways. Secondly, the common methods of existing conservation decision-making include: decision tree, decision matrix method, fuzzy mathematics and analytic hierarchy process, etc., which have shortcomings such as poor portability and large variability, and are greatly affected by regional characteristics and subjective experience. Simulating the complexity of real-world decision problems ${ }^{[5-7]}$. Third, the existing conservation decision model does not incorporate the utility of the traveler as a consideration in decision analysis.

In view of this, from the perspective of improving the economic benefits of highway enterprises, this paper integrates the utility of travellers into the analysis of highway maintenance plan decision-making, and uses Stackelberg under the assumption that their interests are maximized. Game theory analyzes the interaction process between the management department and the traveler, and establishes a model. After describing the related problems, the sensitivity-based heuristic algorithm ${ }^{[8]}$ is used to maintain the two highways with the number $\mathrm{N}$ of travellers. The decision model performs the calculation and obtains the corresponding decision table. Finally, based on the different decision results in the decision table, the general process of multiple highway maintenance decision analysis with the number of travellers is given. The process has broad applicability and proposes a new way for the decision-making of maintenance planning for expressway operators.

\section{Methodology}

\subsection{Decision making of Expressway maintenance Plan under different Road conditions for travelers}

If the traveler (driver, vehicle, traffic volume) arrives from Area A and there are multiple highways to choose from; the traveler will choose the route with the maximum utility, and the road with poor quality will affect the traveler's Driving effectiveness and cost; the management department will maximize the difference between toll revenue and maintenance cost when making maintenance plans for the line, and the distribution of traffic flow on different lines will affect the total toll revenue and maintenance cost of the manager.

There is a dynamic decision-making process between the traveler and the management department: 1 the manager selects the road section for the big and medium repair; (2) the traveler decides which road to drive according to the road condition; (3) the manager adjusts according to the usage of each line (the distribution of the traffic flow) Maintenance plan; (4) travellers adjust the driving route according to the maintenance and maintenance situation. The rational and stable strategy is gradually formed after repeated and repeated games.

This problem constitutes a typical Stackelberg game problem. The main players are management departments and travellers. The management department is the leader and the traveler is the follower. The management maximizes its own payment (the difference between income and cost) through the adjustment of the maintenance plan, and the traveler maximizes his payment (the difference between driving utility and cost) through the choice of road.

\subsection{Establishment of Expressway maintenance Plan Decision-making Model related to Travelers}

The main factors that influence the strategy choice of traveler and management include the benefits (utility or income) and cost in the course of road selection. For ease of analysis, the following assumptions are made:

Hypothesis 1: The traveler has $M$ highways to choose from A to B. If there are other roads, the other roads can be regarded as a whole $M+1$ roads, and from A to $\mathrm{B}$. The total traffic volume is $N$. Travelers and managers are rational, that is, they are seeking to maximize the difference between revenue and cost. For travellers, the probability of choosing the $i(i=1,2, \ldots, M)$ road is $x_{i}$; the utility obtained by driving on the road with the current value of the road is $w_{i}$; The additional net utility obtained by driving on the road after the service level is improved

ISSN: 0010-8189 
is $p_{i}$; the toll of the $i$-th road is $c_{i}$.

Hypothesis 2: The utility $w_{i}$ is the service reward that the traveler receives after paying the travel cost $c_{i}$. When the road condition drops, the $w_{i}$ decreases, and the highway department maintains the funds through the investment, so that the road surface condition is excellent, and the travel is compensated. Loss of utility. Therefore, after the road is repaired, the technical condition is improved so that the traveler obtains additional net utility $p_{i}$, and the utility $\left(w_{i}+p_{i}\right)$ is the maximum return of the service enjoyed by the traveler after paying the road fee.

Hypothesis 3: Let $y_{i}$ denote the management decision variable of the $i$-th road of the management department, and take the value of 0,1 variable, that is, if the $i$-th road large-scale maintenance plan is arranged, $y_{i}=1$, otherwise $y_{i}$ $=0$; The toll income of the $i$-th road is $N x_{i} c_{i}$; the normal operating cost of the road is Oi; if the maintenance of the $i$-th road is maintained, the maintenance cost is $O_{i}$, and the maintenance cost is limited by the total income percentage $T$.

According to the above assumptions, the maintenance plan decision model of $M$ highways with traffic volume $N$ is as follows:

$$
\begin{array}{r}
\text { Management: }(U): \max \sum_{i=1}^{M}\left[y_{i}\left(N x_{i} c_{i}-O_{i}-Q_{i}\right)+\left(1-y_{i}\right)\left(N x_{i} c_{i}-O_{i}\right)\right] \\
\text { s.t }: y_{i}=0 \text { or } 1, \sum_{i=1}^{M} y_{i} Q_{i} \leq T, y_{i}=F\left(x_{1}, x_{2}, \mathrm{~K}, x_{M}\right) . \\
\text { Traveler: } \quad(L): \max \sum_{i=1}^{M} x_{i}\left[y_{i}\left(w_{i}+p_{i}-c_{i}\right)+\left(1-y_{i}\right)\left(w_{i}-c_{i}\right)\right] \\
\text { s.t }: 0 \leq x_{i} \leq 1, \sum_{i=1}^{M} x_{i}=1, p_{i}=g\left(Q_{i}\right), x_{i}=G\left(y_{1}, y_{2}, \ldots, y_{M}\right) .
\end{array}
$$

In formulas (1) and (2), $F, G$ :is a function of interaction between travelers and managers in decision-making, and $g$ is the additional effect function of maintenance congestion on travelers.

In this paper, we first analyze the decision of two highways $(M=2)$ in special cases, and then extend the conclusions to the general situation of multiple highways $(M \geq 3)$, and give the corresponding analysis ideas.

\section{Calculation and Discussion on the Solution of Maintenance Plan Decision-Making Model}

\subsection{Model solving operation}

In order to simplify the analysis of the problem, there are two expressways to choose from from the A to the B. The traffic volume is $N$. The extra net utility that the traveler gets in the case of road maintenance (the more the cost into the maintenance) The more utility you get) is a linear function of maintenance costs (costs invested in road maintenance): $p_{i}=g\left(Q_{i}\right)=k_{i} Q_{i}(0<k<1)$. The maintenance plan decision models for the two highways are as follows:

Management: $\max _{y_{1}, y_{2}} U=\left[N\left(x_{1} c_{1}+x_{2} c_{2}\right)-\left(O_{1}+O_{2}\right)-\left(y_{1} Q_{1}+y_{2} Q_{2}\right)\right]$
s.t $: y_{i}=0, \quad \sum_{i=1}^{2} y_{i} Q_{i} \leq T$. 
Traveler:

$$
\begin{gathered}
\max _{x_{1}, x_{2}} L=\left[x_{1}\left(w_{1}+y_{1} k_{1} Q_{1}-c_{1}\right)+x_{2}\left(w_{2}+y_{2} k_{2} Q_{2}-c_{2}\right)\right] \\
\text { s.t }: 0 \leq x_{i} \leq 1, \quad \sum_{i=1}^{2} x_{i}=1 .
\end{gathered}
$$

This bi-level programming problem is an NP-hard problem, and there is no polynomial solving algorithm [9], usually using a heuristic algorithm [10]. In this paper, the sensitivity analysis is used to find the derivative relationship between variables, and then the Taylor expansion is used to approximate the response function, that is, the heuristic algorithm based on sensitivity analysis (SAB) [10-12], and finally the reaction function is substituted into the objective function. According to the Stackelberg game solving process, the optimal decision of the pedestrian and the manager is obtained.

Assuming that $\mathrm{Y}^{(0)}$ is the initial value of the management maintenance choice, it is possible to solve the path selection result of the traveller under the maximization of his own interests under this initial value $X^{*}\left(\mathrm{Y}^{(0)}\right)$, and through sensitivity analysis, the derivative relationship between the probability of different road choices and the maintenance choice of management department is obtained. $\frac{\partial x}{\partial y}$, then the Taylor expansion which reflects the function is approximately $x(y) \approx X^{*}\left(\mathrm{Y}^{(0)}\right)+\frac{\partial x}{\partial y}\left(y-\mathrm{Y}^{(0)}\right)$, this function is a concrete form of the response function.

By planning (2):

(I): if $w_{1}-c_{1} \geq w_{2}-c_{2}$, then $\left(y_{1}, y_{2} ; \mathbf{x}_{1}, \mathbf{x}_{2}\right)=(0,0 ; 1,0)$ for the initial solution of the program, according to the Taylor expansion:

$$
\begin{gathered}
x_{1} \approx 1+\frac{\partial x_{1}}{\partial y_{1}} y_{1} ; x_{1} \approx 1+\frac{\partial x_{1}}{\partial y_{2}} y_{2} ; x_{2} \approx \frac{\partial x_{2}}{\partial y_{1}} y_{1} ; x_{2} \approx \frac{\partial x_{2}}{\partial y_{2}} y_{2} \\
\rho\left(v_{i}, V_{i P}\right)=\left|v_{i}-\frac{1}{2}\left(a_{P i}+b_{P i}\right)\right|-\frac{1}{2}\left(b_{P i}-a_{P i}\right)= \begin{cases}a_{P i}-v_{i} & v_{i} \leq \frac{a_{P i}+b_{P i}}{2} \\
v_{i}-b_{P i} & v_{i} \phi \frac{a_{P i}+b_{P i}}{2}\end{cases}
\end{gathered}
$$

Substitute in $L$ :

$$
\begin{gathered}
L=k_{1} N c_{1} x_{1}^{2}+k_{2} N c_{2} x_{2}^{2}+\left(k_{1} c_{2}+k_{2} c_{1}\right) N x_{1} x_{2}+\left(w_{1}-c_{1}-k_{1} N c_{1}\right) x_{1}+\left(w_{2}-c_{2}-k_{2} N c_{1}\right) x_{2} \\
\left\{\begin{array}{l}
\frac{\partial L}{\partial x_{1}}=2 k_{1} N c_{1} x_{1}+\left(k_{1} c_{2}+k_{2} c_{1}\right) N x_{2}+\left(w_{1}-c_{1}-k_{1} N c_{1}\right)=0 \\
\frac{\partial L}{\partial x_{2}}=2 k_{2} N c_{2} x_{2}+\left(k_{1} c_{2}+k_{2} c_{1}\right) N x_{1}+\left(w_{2}-c_{2}-k_{2} N c_{1}\right)=0
\end{array}\right.
\end{gathered}
$$

Also for the factor $\sum_{i=1}^{2} x_{i}=1$ we get:

ISSN: 0010-8189 


$$
\begin{aligned}
& \left\{\begin{array}{l}
x_{1}=\frac{c_{1}-w_{1}+k_{1} N c_{1}-k_{2} N c_{1}-k_{1} N c_{2}}{N\left(2 k_{1} c_{1}-k_{1} c_{2}-k_{2} c_{1}\right)}=\frac{2 k_{2} N c_{2}+w_{2}-c_{2}-k_{2} N c_{1}}{N\left(2 k_{2} c_{2}-k_{1} c_{2}-k_{2} c_{1}\right)} \\
x_{2}=\frac{k_{1} N c_{1}+w_{1}-c_{1}}{N\left(2 k_{1} c_{1}-k_{1} c_{2}-k_{2} c_{1}\right)}
\end{array}\right. \\
& y_{i}=0 、 1, \quad \sum_{i=1}^{2} y_{i} Q_{i} \leq T, \quad 0 \leq x_{i} \leq 1, \quad \sum_{i=1}^{2} x_{i} \leq 1, \quad w_{1}-c_{1} \geq w_{2}-c_{2} \\
& \left\{\begin{array}{l}
c_{2}-c_{1}=0, k_{1} N c_{1}+w_{1}-c_{1}=0 \\
\frac{\left(c_{2}-c_{1}\right)}{Q_{1}} * \frac{k_{1} N c_{1}+w_{1}-c_{1}}{2 k_{1} c_{1}-k_{1} c_{2}-k_{2} c_{1}}=1, \frac{\left(c_{2}-c_{1}\right)}{Q_{2}} * \frac{k_{1} N c_{1}+w_{1}-c_{1}}{2 k_{1} c_{1}-k_{1} c_{2}-k_{2} c_{1}}=1 \\
\frac{\left(k_{1} N c_{1}+w_{1}-c_{1}\right)\left(c_{2}-c_{1}\right)}{2 k_{1} c_{1}-k_{1} c_{2}-k_{2} c_{1}} \leq \frac{T}{2}, 0 \leq \frac{k_{1} N c_{1}+w_{1}-c_{1}}{N\left(2 k_{1} c_{1}-k_{1} c_{2}-k_{2} c_{1}\right)} \leq 1 \\
\frac{c_{1}-w_{1}+k_{1} N c_{1}-k_{2} N c_{1}-k_{1} N c_{2}}{N\left(2 k_{1} c_{1}-k_{1} c_{2}-k_{2} c_{1}\right)}=\frac{2 k_{2} N c_{2}+w_{2}-c_{2}-k_{2} N c_{1}}{N\left(2 k_{2} c_{2}-k_{1} c_{2}-k_{2} c_{1}\right)} \\
w_{1}-c_{1} \geq w_{2}-c_{2}
\end{array}\right.
\end{aligned}
$$

(II): if $w_{1}-c_{1}<w_{2}-c_{2}$, then $\left(y_{1}, y_{2} ; x_{1}, x_{2}\right)=(0,0 ; 0,1)$ can be the initial solution of the program, and then according to the Taylor expansion:

Substitute in $L$ :

$$
\begin{aligned}
& x_{1} \approx \frac{\partial x_{1}}{\partial y_{1}} y_{1} ; x_{1} \approx \frac{\partial x_{1}}{\partial y_{2}} y_{2} ; x_{2} \approx 1+\frac{\partial x_{2}}{\partial y_{1}} y_{1} ; x_{2} \approx 1+\frac{\partial x_{2}}{\partial y_{2}} y_{2} \\
& \left\{\begin{array} { l } 
{ \frac { \partial U } { \partial y _ { 1 } } = N ( \frac { \partial x _ { 1 } } { \partial y _ { 1 } } c _ { 1 } + \frac { \partial x _ { 2 } } { \partial y _ { 1 } } c _ { 2 } ) - Q _ { 1 } = 0 } \\
{ \frac { \partial U } { \partial y _ { 2 } } = N ( \frac { \partial x _ { 1 } } { \partial y _ { 2 } } c _ { 1 } + \frac { \partial x _ { 2 } } { \partial y _ { 2 } } c _ { 2 } ) - Q _ { 2 } = 0 }
\end{array} \Rightarrow \left\{\begin{array}{l}
y_{1}=\frac{N}{Q_{1}}\left(x_{1} c_{1}+x_{2} c_{2}-c_{2}\right) \\
y_{2}=\frac{N}{Q_{2}}\left(x_{1} c_{1}+x_{2} c_{2}-c_{2}\right)
\end{array}\right.\right.
\end{aligned}
$$

$$
\begin{aligned}
& \qquad L=k_{1} N c_{1} x_{1}^{2}+k_{2} N c_{2} x_{2}^{2}+\left(k_{1} c_{2}+k_{2} c_{1}\right) N x_{1} x_{2}+\left(w_{1}-c_{1}-k_{1} N c_{2}\right) x_{1}+\left(w_{2}-c_{2}-k_{2} N c_{2}\right) x_{2} \\
& \text { For factor } \sum_{i=1}^{2} x_{i}=1 \text {, we get: }
\end{aligned}
$$

$$
\begin{aligned}
&\left\{\begin{array}{l}
x_{1}=\frac{c_{1}-w_{1}+k_{1} N c_{1}-k_{2} N c_{1}-k_{1} N c_{2}}{N\left(2 k_{1} c_{1}-k_{1} c_{2}-k_{2} c_{1}\right)}=\frac{k_{2} N c_{2}+w_{2}-c_{2}}{N\left(2 k_{2} c_{2}-k_{1} c_{2}-k_{2} c_{1}\right)} \\
x_{2}=\frac{k_{1} N c_{1}+w_{1}-c_{1}}{N\left(2 k_{1} c_{1}-k_{1} c_{2}-k_{2} c_{1}\right)}
\end{array}\right. \\
& \Rightarrow\left\{\begin{array}{l}
y_{1}=\frac{\left(c_{1}-c_{2}\right)}{Q_{1}} * \frac{k_{2} N c_{2}+w_{2}-c_{2}}{2 k_{2} c_{2}-k_{1} c_{2}-k_{2} c_{1}} \\
y_{2}=\frac{\left(c_{1}-c_{2}\right)}{Q_{2}} * \frac{k_{2} N c_{2}+w_{2}-c_{2}}{2 k_{2} c_{2}-k_{1} c_{2}-k_{2} c_{1}},
\end{array}\right. \\
& y_{i}=0 、 1, \quad \sum_{i=1}^{2} y_{i} Q_{i} \leq T, \quad 0 \leq x_{i} \leq 1, \quad \sum_{i=1}^{2} x_{i} \leq 1, \quad w_{1}-c_{1} \geq w_{2}-c_{2}
\end{aligned}
$$




$$
\Rightarrow\left\{\begin{array}{l}
c_{1}-c_{2}=0 ; k_{2} N c_{2}+w_{2}-c_{2}=0 \\
\frac{\left(c_{1}-c_{2}\right)}{Q_{1}} * \frac{k_{2} N c_{2}+w_{2}-c_{2}}{2 k_{2} c_{2}-k_{1} c_{2}-k_{2} c_{1}}=1 ; \frac{\left(c_{1}-c_{2}\right)}{Q_{2}} * \frac{k_{2} N c_{2}+w_{2}-c_{2}}{2 k_{2} c_{2}-k_{1} c_{2}-k_{2} c_{1}}=1 \\
\frac{\left(k_{2} N c_{2}+w_{2}-c_{2}\right)\left(c_{1}-c_{2}\right)}{2 k_{2} c_{2}-k_{1} c_{2}-k_{2} c_{1}} \leq \frac{T}{2} \\
0 \leq \frac{k_{1} N c_{1}+w_{1}-c_{1}}{N\left(2 k_{1} c_{1}-k_{1} c_{2}-k_{2} c_{1}\right)} \leq 1 \\
\frac{c_{1}-w_{1}+k_{1} N c_{1}-k_{2} N c_{1}-k_{1} N c_{2}}{N\left(2 k_{1} c_{1}-k_{1} c_{2}-k_{2} c_{1}\right)}=\frac{k_{2} N c_{2}+w_{2}-c_{2}}{N\left(2 k_{2} c_{2}-k_{1} c_{2}-k_{2} c_{1}\right)} \\
w_{1}-c_{1}<w_{2}-c_{2}
\end{array}\right.
$$

(III):

(1) If the decision of the management department is $\left(y_{1}, y_{2}\right)=(0,0), \quad w_{1}-c_{1} \geq w_{2}-c_{2}$, the optimal decision of the traveler is $\left(x_{1}, x_{2}\right)=(1,0)$, at this time $U_{11}=N c_{1}-O_{1}-O_{2} ; w_{1}-c_{1}<w_{2}-c_{2}$, the optimal decision of the traveler is $\left(x_{1}, x_{2}\right)$ $=(0,1)$, at which time $U_{12}=\mathrm{Nc}_{2}-\mathrm{O}_{1}-\mathrm{O}_{2}$

(2) If the management decision is $\quad\left(y_{1}, y_{2}\right)=(1,1), w_{1}-c_{1}+k_{1} Q_{1} \geq w_{2}-c_{2}+k_{2} Q_{2}$, the traveler's optimal decision is $\quad\left(x_{1}, x_{2}\right)$ $=(1,0)$, this When $U_{21}=N c_{1}-O_{1}-O_{2}-Q_{2}$; when $w_{1}-c_{1}<w_{2}-c_{2}+k_{2} Q_{2}$, the optimal decision of the traveler is $\left(x_{1}, x_{2}\right)$ $=(0,1)$, and $U_{22}=N c_{2}-O_{1}-O_{2}-Q_{2}$.

(3) If the management decision is $\left(y_{1}, y_{2}\right)=(1,0), w_{1}-c_{1}+k_{1} Q_{1} \geq w_{2}-c_{2}$, the traveler's optimal decision is $\left(x_{1}, x_{2}\right)=(1,0)$, this When $U_{31}=N c_{1}-O_{1}-O_{2}-Q_{1}$; when $w_{1}-c_{1}<w_{2}-c_{2}+k_{2} Q_{2}$, the optimal decision of the traveler is $\quad\left(x_{1}, x_{2}\right)=(0,1)$, at this time $U_{32}=\mathrm{Nc}_{2}-\mathrm{O}_{1}-\mathrm{O}_{2}-Q_{1}$.

(4) If the management decision is $\left(y_{1}, y_{2}\right)=(1,1), w_{1}-c_{1}+k_{1} Q_{1} \geq w_{2}-c_{2}+k_{2} Q_{2}$, the traveler's optimal decision is $\left(x_{1}, x_{2}\right)$ $=(1,0)$ At this time, $U_{41}=N c_{1}-O_{1}-O_{2}-Q_{1}-Q_{2} ; w_{1}-c_{1}+k_{1} Q_{1}<w_{2}-c_{2}+k_{2} Q_{2}$, the optimal decision of the traveler is $\left(x_{1}, x_{2}\right)$ $=(0,1), U_{42}=N c_{2}-O_{1}-O_{2}-Q_{1}-Q_{2}$.

Through calculation, the comprehensive I, II, and III are selected to obtain the travel group selection route and the decision of the two highway maintenance plans, as shown in Table 1.

Table 1 Decision-making tables of route selection and two highway maintenance plans for travel groups

\begin{tabular}{|c|c|}
\hline Decision result & Decision condition \\
\hline $\begin{array}{l}\text { Road A is maintained, road } \\
B \text { is maintained }\end{array}$ & $\begin{aligned} \text { if: (1) } Q_{1} & \left.=Q_{2}=\frac{\left(c_{2}-c_{1}\right)\left(k_{1} N c_{1}+w_{1}-c_{1}\right)}{2 k_{1} c_{1}-k_{1} c_{2}-k_{2} c_{1}},\left(c_{1}<c_{2}\right) \text { and meet ( } \mathrm{a}\right) \text {; } \\
\text { (2) } Q_{1} & \left.=Q_{2}=\frac{\left(c_{1}-c_{2}\right)\left(k_{2} N c_{2}+w_{2}-c_{2}\right)}{2 k_{2} c_{2}-k_{1} c_{2}-k_{2} c_{1}}, \quad\left(c_{1}>c_{2}\right) \text { and meet ( }\right)\end{aligned}$ \\
\hline $\begin{array}{l}\text { Road A is maintained, road } \\
B \text { is not maintained }\end{array}$ & (3) if: $w_{1}-c_{1}<w_{2}-c_{2}<w_{1}-c_{1}+k_{1} Q_{1}$ and $N c_{1}-N c_{2}-Q_{1} \geq 0$ \\
\hline $\begin{array}{l}\text { Road A is not maintained, } \\
\text { road B is maintained }\end{array}$ & (4) if: $w_{2}-c_{2}<w_{1}-c_{1}<w_{2}-c_{2}+k_{2} Q_{2}$ and $N c_{2}-N c_{1}-Q_{2} \geq 0$ \\
\hline $\begin{array}{l}\text { A, B Two roads not to be } \\
\text { repaired for the time being }\end{array}$ & 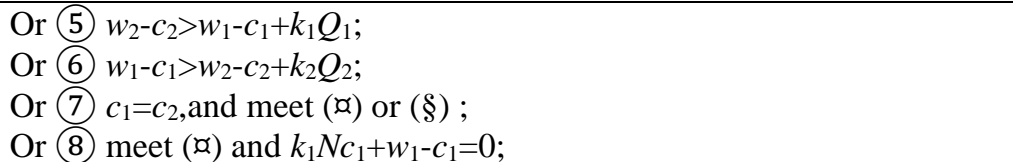 \\
\hline
\end{tabular}

ISSN: 0010-8189 
$(a)$

$$
\begin{aligned}
& \left\{\begin{array}{l}
\frac{\left(k_{1} N c_{1}+w_{1}-c_{1}\right)\left(c_{2}-c_{1}\right)}{2 k_{1} c_{1}-k_{1} c_{2}-k_{2} c_{1}} \leq \frac{T}{2}, 0 \leq \frac{k_{1} N c_{1}+w_{1}-c_{1}}{N\left(2 k_{1} c_{1}-k_{1} c_{2}-k_{2} c_{1}\right)} \leq 1 \\
\frac{c_{1}-w_{1}+k_{1} N c_{1}-k_{2} N c_{1}-k_{1} N c_{2}}{N\left(2 k_{1} c_{1}-k_{1} c_{2}-k_{2} c_{1}\right)}=\frac{2 k_{2} N c_{2}+w_{2}-c_{2}-k_{2} N c_{1}}{N\left(2 k_{2} c_{2}-k_{1} c_{2}-k_{2} c_{1}\right)} \\
w_{1}-c_{1} \geq w_{2}-c_{2}
\end{array}\right. \\
& \left\{\begin{array}{l}
\frac{\left(k_{2} N c_{2}+w_{2}-c_{2}\right)\left(c_{1}-c_{2}\right)}{2 k_{2} c_{2}-k_{1} c_{2}-k_{2} c_{1}} \leq \frac{T}{2}, 0 \leq \frac{k_{1} N c_{1}+w_{1}-c_{1}}{N\left(2 k_{1} c_{1}-k_{1} c_{2}-k_{2} c_{1}\right)} \leq 1 \\
\frac{c_{1}-w_{1}+k_{1} N c_{1}-k_{2} N c_{1}-k_{1} N c_{2}}{N\left(2 k_{1} c_{1}-k_{1} c_{2}-k_{2} c_{1}\right)}=\frac{k_{2} N c_{2}+w_{2}-c_{2}}{N\left(2 k_{2} c_{2}-k_{1} c_{2}-k_{2} c_{1}\right)} \\
w_{1}-c_{1}<w_{2}-c_{2}
\end{array}\right.
\end{aligned}
$$

\subsection{Discussion on maintenance Plan decision of two Expressway}

Two highway maintenance plan decision tables with traffic $N$ above may be discussed as follows:

Discussion 1: the condition of arranging the maintenance of two roads at the same time is that the investment and scale of the maintenance of the two roads are equal, otherwise the two roads will lose their original balance.

Discuss the conditions in (I) to maintain both roads at the same time:

$$
y_{1}=\frac{\left(c_{2}-c_{1}\right)}{Q_{1}} * \frac{k_{1} N c_{1}+w_{1}-c_{1}}{2 k_{1} c_{1}-k_{1} c_{2}-k_{2} c_{1}}=1, y_{2}=\frac{\left(c_{2}-c_{1}\right)}{Q_{2}} * \frac{k_{1} N c_{1}+w_{1}-c_{1}}{2 k_{1} c_{1}-k_{1} c_{2}-k_{2} c_{1}}=1
$$

Then there is $Q_{1}=Q_{2}$, In the same way, under the condition that II is satisfied, there are $Q_{1}=Q_{2}$, then discuss a proof.

Discussion 2: A necessary condition for the simultaneous maintenance of two roads is that the cost of maintenance invested is less than the difference in road fee income $\left|N c_{1}-N c_{2}\right|$. Because of the conditions ( $\mathrm{a}$ ) (§) so: $0 \leq \frac{\left(k_{i} N c_{i}+w_{i}-c_{i}\right)}{N\left(2 k_{i} c_{i}-k_{j} c_{i}-k_{i} c_{j}\right)} \leq 1 \quad, \quad$ in $\quad i, \quad j=1,2$, and $\quad i \neq j \quad$. Decision conditions(1)(2), so $Q_{1}=Q_{2}=\left|\frac{\left(c_{1}-c_{2}\right)\left(k_{i} N c_{i}+w_{i}-c_{i}\right)}{2 k_{i} c_{i}-k_{j} c_{i}-k_{i} c_{j}}\right| \leq\left|N c_{1}-N c_{2}\right|$.

The income of the two roads is related to the volume of traffic $\left|N c_{1}-N c_{2}\right|$ difference between traffic volume and traffic volume $\left|N_{1}-N_{2}\right|$ has equivalent recitation, the maintenance of two roads at the same time is bound to affect the respective traffic volume. Therefore, it is concluded that the cost input of the two roads is based on the difference of their respective income or traffic volume, and it is not suitable for the maintenance to affect the relative equilibrium of the road network. 
Volume 2021, No. 3

Discussion 3: If the net utility of one road to the traveler is less than that of the other road, and the net utility of this road after maintenance is greater than that of the other road, and satisfied $N c_{1}-N c_{2}-Q_{1} \geq 0$, then this road can be repaired.

Decision conditions from Table 1 (3), $w_{1}-c_{1}<w_{2}-c_{2}<w_{1}-c_{1}+k_{1} Q_{1}$, we can know:

a) When the two roads are not scheduled for maintenance, the travelers are unwilling to take the first road and all choose the second one. The effectiveness of the management is as follows $\mathrm{U}_{1}=\mathrm{Nc}_{2}-\mathrm{O}_{1}-\mathrm{O}_{2}$.

$b$ ) If all travelers choose to travel on the first road for maintenance and repair, the management's utility is $U_{2}=N c_{1}-O_{1}-O_{2}-Q_{1}$.

c) If all travelers choose to travel on the second road where maintenance and repair are carried out, the effect of the management is as follows $\mathrm{U}_{3}=\mathrm{Nc}_{2}-\mathrm{O}_{1}-\mathrm{O}_{2}-Q_{2}$.

d) If both roads are maintained and maintained, the traveler will either take the second road, at this time $U_{4}=N_{2}-O_{1}-O_{2}-Q_{1}-Q_{2}$ The traveler either took the first path, at this time $U_{4}=N c_{1}-O_{1}-O_{2}-Q_{1}-Q_{2}$.

Besides $N c_{1}-N c_{2}-Q_{1} \geq 0$, we can know $U_{2} \geq U_{1} \geq U_{3}, U_{2} \geq U_{4}$, that is, in this case, the management's best decision is to maintain only the first road. In the same way, $w_{2}-c_{2}<w_{1}-c_{1}<w_{2}-c_{2}+k_{2} Q_{2}$ and $N c_{2}-N c_{1}-Q_{2} \geq 0$, it is best for management to overhaul only the second road.

Discussion 4: If the net utility of one road to the traveler is greater than that of the other road after maintenance, the optimal decision is that the maintenance of both roads should not be arranged for the time being. From $w_{2}-c_{2}>w_{1}-c_{1}+k_{1} Q_{1}$, we can know:

a) In the case of temporary maintenance and repair, the traveller will take the second path, where the management's utility is $U_{1}=\mathrm{Nc}_{2}-\mathrm{O}_{1}-\mathrm{O}_{2}$

$b$ ) If the first road is repaired, the traveller will still take the second one, and the management's utility will be $U_{2}=N c_{2}-O_{1}-O_{2}-Q_{1}$

c) If the second road is repaired, the traveller will still take the second one, and the management's utility will be $U_{3}=\mathrm{Nc}_{2}-\mathrm{O}_{1}-\mathrm{O}_{2}-Q_{2}$

d) If both roads are repaired at the same time, the traveller will still take the second path, and the management's utility will be $U_{4}=N_{c_{2}-} O_{1}-O_{2}-Q_{1}-Q_{2}$. At this moment $U_{1}>U_{2}, U_{1}>U_{3}, U_{1}>U_{4}$, the optimal maintenance decision in this case is that maintenance plans are not scheduled for either road for the time being. Similarly, when $w_{1}-c_{1}>w_{2}-c_{2}+k_{2} Q_{2}$, the optimal maintenance decision is also a temporary suspension of maintenance.

Discussion 5: If the tolls of two roads are equal, the optimal decision is not to repair the two roads at the same time. Because when $c_{2}=c_{1}$ there are conditions following:

a) In the absence of maintenance, the traveler will choose either path, where the management's utility is $U 1=N c_{1}-O_{1}-O_{2}$.

$b$ ) If the first road is maintained, the traveller will choose the first one, and the management's utility will be $U 2=N c_{1}-O_{1}-O_{2}-Q_{1}$.

c) If the second road is maintained, the traveler will take the second one, and the management will have the effect of $U_{3}=N c_{1}-O_{1}-O_{2}-Q_{2}$.

ISSN: 0010-8189 
d) If both roads are maintained, regardless of which route the traveler takes, the management's utility is $U_{4}=N c_{1}-O_{1}-O_{2}-Q_{1}-Q_{2}$. At this moment $U_{1}>U_{2}, U_{1}>U_{3}$ and $U_{1}>U_{4}$, the optimal maintenance decision in this case is that neither road is need to be maintained.

\subsection{Discussion on maintenance Plan decision of multiple Expressways Management at the same time}

The maintenance plan decision of several highways is the extension of the maintenance plan decision of two highways. The analysis process is similar. Because of the limited space, the analysis process of the maintenance decision of $M$ (multiple) expressway with traffic volume $N$ is only given as follows:

The first step, according to the actual background of the decision-making problem, the maintenance decision model of $M$ (multiple) highways with $N$ traffic volume is established, which is similar to model one. The parameters in the model are given by the practical meaning of the problem.

The second step, the model is usually a bilevel programming problem. If it is a NP-hard problem, the heuristic algorithm is used to solve the optimal equilibrium solution. First of all, assume that $\mathrm{Y}^{(0)}$ is for management to maintain the initial value of the choice, then under this initial value can be solved by the traveler in their own interests under the maximum of the road selection results $\mathrm{X}^{*}\left(\mathrm{Y}^{(0)}\right)$, and through sensitivity analysis, the derivative relationship between the probability of different road choices and the maintenance choice of management department is obtained. $\frac{\partial x}{\partial y}$ then the Taylor expansion which reflects the function is approximately $x(y) \approx \mathrm{x}^{*}\left(\mathrm{Y}^{(0)}\right)+\frac{\partial x}{\partial y}\left(y-\mathrm{Y}^{(0)}\right)$, then the reaction function is substituted into the objective function and the equilibrium solution of the traveler and manager is obtained according to the Stackelberg game.

In the third step, if the equilibrium solution obtained in the second step can satisfy the above conditions, the analysis step ends, and the equilibrium solution is the final maintenance decision sought. If the equilibrium solution obtained in the second step does not meet the conditions, list all possible maintenance plan decisions of the management department, and find out the benefits that the management has obtained under the choice of the maximum benefit of the traveler. The management department has the greatest interest. The maintenance decision is the optimal planning decision that the management should choose.

\section{Conclusion}

This paper explores the decision-making problem of highway managers choosing roads for maintenance, taking the utility of travellers as a factor and adding them to decision analysis, using the Stackelberg game process to analyze the interaction process between management and travellers, managing services and travelers, and build a model to describe the problem. Using the sensitivity-based heuristic algorithm, this paper solves the maintenance decision model of two expressways, and finally draws the relevant decision table. The conclusions are as follows: (1) The conditions for managers to arrange intermediate or major repairs for both roads are as follows: The investment and scale of the maintenance of the two roads are comparable; Or its maintenance cost is based on the difference of income or traffic volume between the two routes, and it is not suitable to affect the relative equilibrium of the road network because of simultaneous maintenance; (2) If the net utility of one road to the traveler is less than that of the other road, and the net utility of this road after maintenance is greater than that of the other road, then the manager may arrange for the maintenance of the road; (3) If the net utility of one road to the traveler is greater than that of the other road after maintenance, then the optimal decision of the manager is not to arrange maintenance for both roads for the time being; (4) If the tolls of the two roads are equal, the optimal decision of

ISSN: 0010-8189 
the manager is not to arrange for the maintenance of the same section of the two roads at the same time; (5) Using the same method, the maintenance decision model of multiple highways can be solved.

\section{Acknowledgements}

This paper is supported by the young innovative talents project of Guangdong ordinary universities (2017WQNCX066).

\section{References}

[1] Xiao-Yan Z, Yin Z, "Analysis and Application of Calculation Formula of Feasibility Gap Subsidy for Expressway PPP Projects,” Journal of Highway \& Transportation Research \& Development, 2018.

[2] Wen-Xin L, Jian-Wei W, Yu-Jun L, "Research on Highway Toll Rates Based on the Cost of Conservation," Journal of Transportation Systems Engineering \& Information Technology, vol. 13, no. 5, pp. 145-149, 2013.

[3] Soltanisobh A, Heaslip K, Stevanovic A, "Evaluation of transportation network reliability during unexpected events with multiple uncertainties," International Journal of Disaster Risk Reduction, vol. 17, pp. 128-136, 2016.

[4] Worm J M, Harten A V, "Model based decision support for planning of road maintenance," Reliability Engineering \& System Safety, vol. 51, no. 3, pp. 305-316, 2017.

[5] G. Hui, X. Zhang, Y. Li, "Developing a weighted reward criterion for the Markov-based decision of road maintenance," Springerplus, vol. 5, no. 1, pp. 1-14, 2016.

[6] Hong-Yan J I, Wang G, Chang-Qing Y U, "Study on the Evaluation and Prediction of Asphalt Pavement Performance Based on the Decision Tree," Highway, pp. 128-136, 2016.

[7] Q.L. Zhu, J. LU, J. LI, "Optimization of Zone Selection of Naval Escort in Pirates Active Areas Based on Stackelberg Games," Journal of Transportation Systems Engineering and Information Technology, vol. 14, no. 6, pp. 152-157, 2014.

[8] Mach F, "Reduction of Optimization Problem by Combination of Optimization Algorithm and Sensitivity Analysis," IEEE Transactions on Magnetics, vol. 52, no. 3, pp. 1-4, 2016.

[9] Sadjadi S J, Asadi H, Sadeghian R, et al. "Retailer Stackelberg game in a supply chain with pricing and service decisions and simple price discount contract," Plos One, vol. 13, no. 4, e0195109, 2018.

[10] Kheirkhah A, Navidi H R, Bidgoli M M, “A bi-level network interdiction model for solving the hazmat routing problem,” International Journal of Production Research, vol. 54, no. 2, pp. 1-13, 2017.

[11] Escobar J W, Linfati R, Toth P, “A two-phase hybrid heuristic algorithm for the capacitated location-routing problem," Computers \& Operations Research, vol. 40, no. 1, pp. 70-79, 2013.

[12] Kritikos M, Ioannou G, "A greedy heuristic for the capacitated minimum spanning tree problem," Journal of the Operational Research Society, pp. 1-13, 2017. 